\title{
Histopathologic Diagnosis of Atypical Primary Vitreoretinal Lymphoma following Enucleation
}

\author{
Arjun B. Sood ${ }^{a, c}$ Steven Yeh ${ }^{a, c}$ Pia Mendoza ${ }^{a-c}$ Hans E. Grossniklaus ${ }^{a, c}$ \\ Departments of ${ }^{\mathrm{a}}$ Ophthalmology and ${ }^{\mathrm{b}}$ Pathology, Emory University, and ${ }^{\mathrm{c} E m o r y}$ Eye Center, Atlanta, Ga., USA
}

\section{Key Words}

Primary vitreoretinal lymphoma $\cdot$ Histopathology

\begin{abstract}
Introduction: Primary vitreoretinal lymphoma (PVRL) is a rare but potentially fatal malignancy. The diagnosis of PVRL remains challenging. Here, we present a case of PVRL with a diagnostic enucleation required to establish the diagnosis. Methods/Case Description: A 58-year-old otherwise healthy woman was referred for evaluation of panuveitis with persistent blurred vision in the left eye. Extensive laboratory workup was negative. On initial evaluation, the patient had visual acuity of no light perception in the left eye. Examination of the left eye revealed diffuse neovascularization of the iris, moderate vitreous debris and no view of the posterior pole. A B-scan ultrasound showed a retinal detachment and extensive subretinal fluid. Enucleation was performed. Results: On gross examination, a white mass was noted under the RPE. Histologic examination showed a mass of large CD20+ lymphocytes under the RPE. Discussion: The histopathologic findings confirmed a diagnosis of large B-cell lymphoma (PVRL). Conclusion: PVRL may masquerade as uveitis, thus leading to a delay in diagnosis and management. A diagnostic enucleation may be useful in blind painful eyes of unknown etiology.

(c) 2016 S. Karger AG, Basel
\end{abstract}

\section{Introduction}

Primary vitreoretinal lymphoma (PVRL) is a rare intraocular malignancy that is characterized by malignant lymphoid cells infiltrating the eye. PVRL is a subset of primary CNS lymphoma (PCNSL) and commonly masquerades as an intermediate or posterior uveitis $[1,2]$. Delay in definitive diagnosis is common and leads to high morbidity [3]. Here, we present a unique case of PVRL diagnosed following enucleation. The patient was subsequently diagnosed with involvement in the fellow eye and CNS relapse. This case report received Emory University IRB exempt status.

\section{Case Report}

A 58-year-old otherwise healthy female was referred to the Uveitis Service at the Emory Eye Center in August 2014 with complaints of blurry vision in her left eye for the past 6 months. She had been diagnosed by the referring provider with panuveitis 3 months prior to presentation. Laboratory investigation was negative for syphilis, tuberculosis, toxoplasmosis, Lyme disease, ACE, Bartonella, ANA and HLA-typing. The patient had been treated with topical and oral steroids, which initially improved inflammation and visual acuity. The patient returned to the referring provider with complaints of worsening vision again in the left

\section{KARGER}

E-Mail karger@karger.com

www.karger.com/oop
(C) 2016 S. Karger AG, Basel

2296-4681/16/0024-0242\$39.50/0
Hans E. Grossniklaus, MD

BT 428 Emory Eye Center 1365 Clifton Road

Atlanta, GA 30322 (USA)

E-Mail ophtheg@emory.edu 

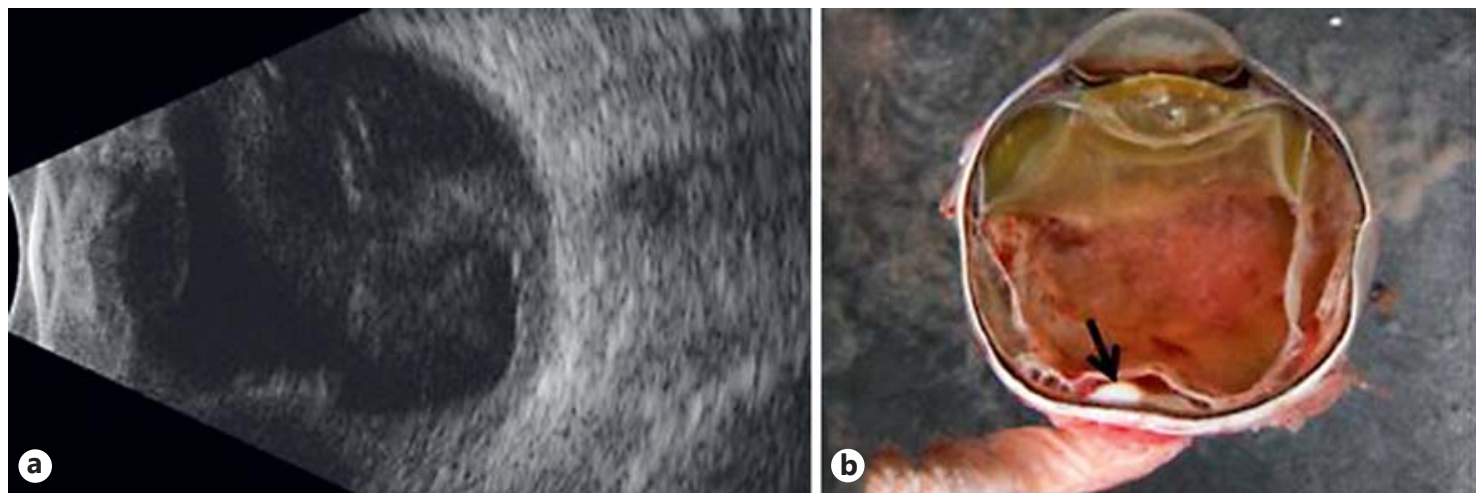

Fig. 1. a B-scan ultrasound of the left eye. There is a retinal detachment with extensive subretinal fluid and hemorrhagic choroidal detachment. b Cut section of the enucleated left eye. The lens has cataractous changes. There is a detached retina with a subretinal white mass.
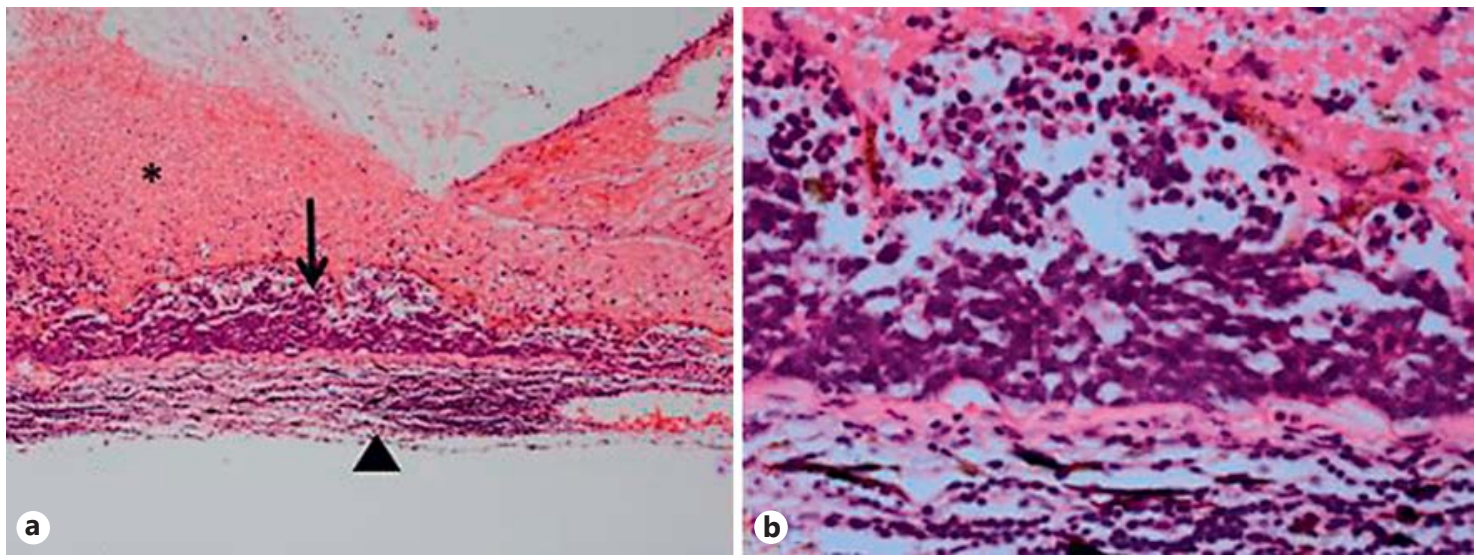

Fig. 2. a The subretinal mass includes an accumulation of large lymphocytes (arrow), which have undergone apoptosis (asterisk) at approximately $100 \mu \mathrm{m}$ internal to Bruch's membrane. There are small lymphocytes (arrowhead) in the choroid. b Large lymphocytes with prominent nucleoli and scanty cytoplasm.
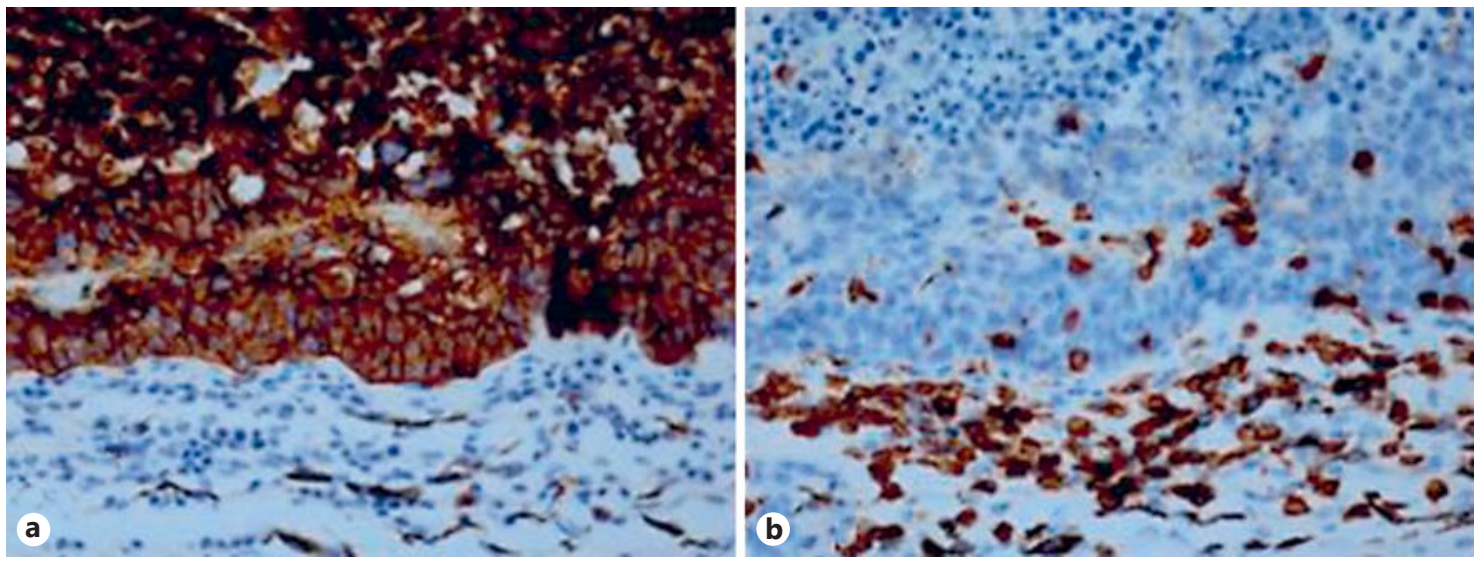

Fig. 3. a The large subretinal lymphocytes stain with CD20. b The small choroidal lymphocytes stain for CD3. Peroxidase anti-peroxidase. $\times 100$. 
eye. Visual acuity was 20/50 in the right eye, 20/200 eccentrically in the left eye. The anterior segment was notable for mild inflammation. Funduscopic examination showed retinal whitening and hemorrhage in the left eye. The right eye was normal. The patient was tapered from oral prednisone and referred to the Emory Eye Center. At initial evaluation, the patient was complaining of total vision loss and pain in the left eye. Visual acuity was 20/100 in the right eye, and no light perception in the left eye. Intraocular pressure was normal in the right eye and $45 \mathrm{~mm} \mathrm{Hg}$ in the left eye. The anterior segment in the left eye showed 360 degrees of neovascularization of the iris with posterior synechiae and trace cell. Funduscopic examination of the right eye showed vitreous opacities, drusen and chronic vascular sheathing. There was no view to the posterior segment in the left eye. B-scan ultrasound was performed on the left eye and showed a retinal detachment with extensive subretinal fluid and hemorrhagic choroidal detachment (fig. 1a).

The patient had developed a blind painful eye secondary to unknown process, and we performed enucleation for both therapeutic and diagnostic purposes.

\section{Pathologic Findings}

External examination of the enucleated eye showed a hazy cornea with keratic precipitates. On gross section, there was cataract formation, blood strands within the retina, a detached necrotic retina and a subretinal white lesion (fig. 1b). Histologic examination showed a mass of lymphocytes located between the retinal pigment epithelium and Bruch's membrane (fig. 2a). These lymphocytes had large nuclei with prominent nucleoli and scanty cytoplasm (fig. 2b). The large, neoplastic lymphocytes stained with CD20, indicating a large B-cell lymphoma (fig. 3a). The small choroidal lymphocytes stained positively with $\mathrm{CD} 3$ indicating a population of reactive T lymphocytes (fig. 3b). Collectively, histopathology of the enucleated left eye confirmed a diagnosis of large B-cell lymphoma (PVRL).

\section{Discussion}

The case presented herein represents an atypical presentation of rapidly progressive and fulminant PVRL diagnosed by enucleation. PVRL represents a subset of PCNSL and is defined as malignant lymphoid cells infiltrating the retina, vitreous and optic nerve [4]. PVRL is a rare ocular malignancy and the exact incidence is unknown. Better data exists for PCNSL with an estimated 1,900 reported cases within the United States in 2007. Intraocular involvement occurs in approximately $20 \%$ of cases and we can therefore estimate there are approxi- mately 300-380 new cases of PVRL a year $[1,4,5]$. There is no racial predilection and PVRL more commonly affects men than women.

PVRL is a masquerade syndrome that mimics intermediate or posterior uveitis and usually affects the elderly (age $>60$ years). Most patients present with complaints of blurred vision and floaters, and ocular findings typically include a severe vitritis with subretinal infiltrates and RPE deposits [1]. Once infectious and inflammatory etiologies are ruled out, these patients commonly receive topical and systemic steroids. Initially, the inflammation and visual symptoms may respond, but the disease progresses, leading to worsening inflammation that is refractory to steroids.

There is a wide differential that must be considered, as PVRL can masquerade as infectious, inflammatory or neoplastic ocular disease [1]. Early diagnosis is critical, as delay can lead to increased morbidity. Once PVRL is suspected, patients should undergo systemic evaluation with neuroimaging and lumbar puncture due to the high incidence of concomitant CNS involvement. If negative, further diagnostic work-up must be pursued $[5,6]$.

Histopathologic identification of malignant lymphoid cells is the gold standard for diagnosis [5]. Tissue can be obtained through various processes including aqueous sampling, vitreous tap, diagnostic vitrectomy, chorioretinal biopsy, and rarely as in our case diagnostic enucleation. Unfortunately, negative results are common, as lymphoma cells undergo rapid degradation following procurement [4]. Additionally, many patients receive preoperative steroid treatment and this contributes to decreased cell recovery. Therefore, it is important to work closely with an experienced pathologist who can perform cytology testing and immunohistochemistry.

This case is unusual due to the method of diagnosis, atypical presentation and fulminant course. AlQahtani et al. [7] reported other atypical presentations of PVRL that share some characteristics. Our patient seems to share these similar characteristics, including a younger age, female gender, presence of anterior chamber inflammation, and a mild vitritis. Our patient and one of the patients reported by AlQahtani et al. both had a rapidly progressive clinical course at the end resulting in no light perception vision [7].

Initial systemic work-up after enucleation was negative, including neuroimaging and lumbar puncture. The fellow eye continued to have low-grade vitreous inflammation and decreased vision. There were no discrete masses or lesions. Therefore, a diagnostic vitrectomy was 
performed and revealed the presence of malignant lymphoid cells in the vitreous sample. The patient underwent local therapy with intravitreal methotrexate, and vision returned to 20/20. Concurrently, the patient was seen by neuro-oncology and undergoing surveillance neuroimaging. In March 2015, repeat MRI showed lesions within the CNS consistent with PCNSL. She was started on systemic high-dose methotrexate, temozolomide and rituximab.

\section{Conclusion}

PVRL often masquerades as intraocular inflammation or uveitis. A delay in diagnosis can lead to significant morbidity, thus diagnostic enucleation of a blind painful eye of unknown etiology should be considered.

\section{Acknowledgement}

This study was supported in part by NIH P3006360, Research to Prevent Blindness, Inc.

\section{Statement of Ethics}

This study is IRB exempt at our institution.

\section{Disclosure Statement}

The authors have no conflicts of interest to disclose.

\section{References}

1 Sagoo, MS, Mehta, H, Swampillai, AJ, et al: Primary intraocular lymphoma. Surv Ophthalmol 2014;59:503-516.

2 Coupland SE, Damato B: Understanding intraocular lymphomas. Clin Experiment Ophthalmol 2008;36:564-578.

3 Davis JL: Intraocular lymphoma: a clinical perspective. Eye (Lond) 2013;27:153-162.
4 Hwang CS, Yeh S, Bergstrom CS: Diagnostic vitrectomy for primary intraocular lymphoma: when, why, how? Int Ophthalmol Clin 2014;54:155-171.

5 Chan CC, Rubenstein JL, Coupland SE, et al: Primary vitreoretinal lymphoma: a report from an International Primary Central Nervous System Lymphoma Collaborative Group symposium. Oncologist 2011;16:1589-1599.
6 Chan CC, Sen HN: Current concepts in diagnosing and managing primary vitreoretinal (intraocular) lymphoma. Discov Med 2013; 15:93-100

7 AlQahtani A, Touitou V, Cassoux N, et al: More than a masquerade syndrome: atypical presentations of vitreoretinal lymphomas. Ocul Immunol Inflamm 2014;22:189-196. 